\title{
Agôn
}

Revue des arts de la scène

$4 \mid 2011$

L'objet

\section{La force des choses}

Animisme et sacralisation de l'objet dans l'univers de la compagnie Deschamps-Makeïeff

\section{Camille Fosse}

\section{(2) OpenEdition}

1 Journals

\section{Édition électronique}

URL : http://journals.openedition.org/agon/1964

DOI : 10.4000/agon.1964

ISSN : 1961-8581

Éditeur

Association Agôn

Référence électronique

Camille Fosse, "La force des choses », Agôn [En ligne], 4 | 2011, mis en ligne le 19 décembre 2011, consulté le 17 avril 2020. URL : http://journals.openedition.org/agon/1964 ; DOI : https://doi.org/ 10.4000/agon.1964 


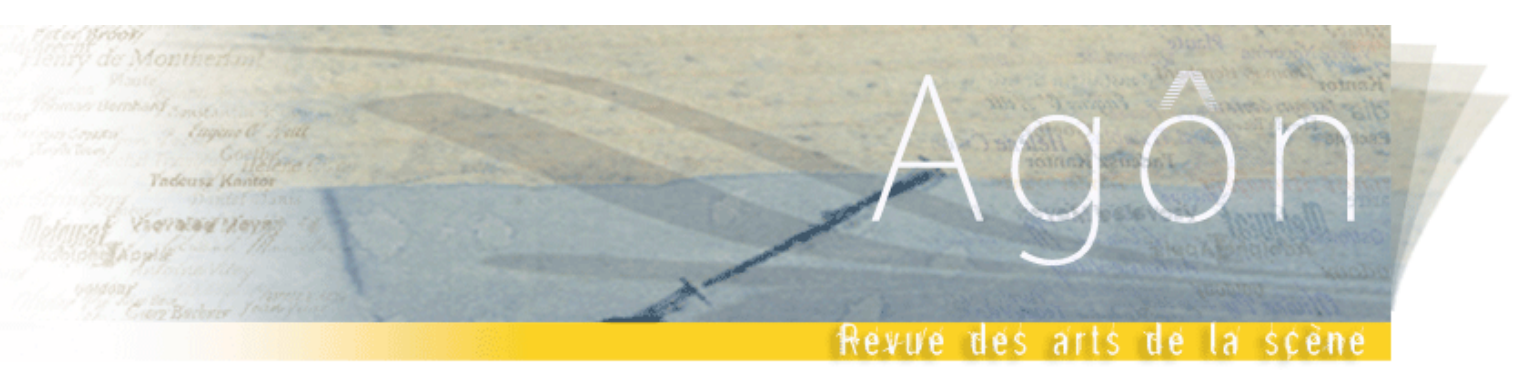

\section{La force des choses}

Animisme et sacralisation de l'objet dans l'univers de la compagnie Deschamps-Makeïeff.

Camille Fosse

\section{Résumé}

L'étude présentée dans cet article explore la manière dont l'objet accède au rang «d'objet sacré » - voire «d'objet martyr »- au sein de la compagnie Deschamps-Makeïeff, par la forme d'un paradigme animiste entretenu par les metteurs en scène. En analysant les aspects de ces investissements affectifs et religieux, nous ferons l'hypothèse selon laquelle l'objet symbolise une certaine vision désenchantée de l'humain et du monde dans les spectacles de la compagnie ainsi que dans les expositions personnelles de Macha Makeïeff. Notre intention sera de questionner le rôle et les fonctions de cette sacralisation de l'objet pour en mesurer les enjeux discursifs et les répercussions identitaires au cœur de l'univers de la compagnie théâtrale.

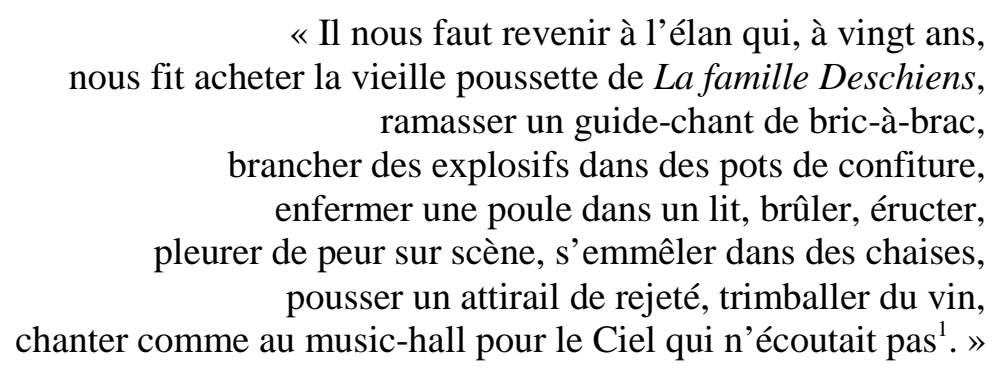

Depuis le milieu des années 1970, la compagnie Deschamps-Makeïeff propose un univers théâtral singulier dans lequel l'objet, loin de se cantonner au simple rôle d'accessoire, occupe une place prépondérante: "Poussettes et chariots, animaux empaillés, bouteilles et flacons, assiettes volantes et verres sauteurs, plantes vertes, gants de toilette, fleurs de plastique, casiers à bouteilles, cabas, papier journal, tue-mouche, bassines, batte de bois,... reviennent comme par fétichisme d'un spectacle à l'autre ${ }^{2}$. » Faire l'inventaire des objets scéniques de la compagnie permet de comprendre « la grammaire visuelle des spectacles ${ }^{3}$ » et bien plus encore. Choses flétries, ordinaires, sans beauté ni grâce particulière, les objets ramassés méticuleusement par Macha Makeïeff pour les besoins de la scène sont considérés

Ibid.

Macha Makeїeff, Deschamps Deschiens, le théâtre de Jérôme Deschamps, Paris, Séguier, 1989, p. 60. 
comme des « rescapés ${ }^{4} »$, des traces d'humanité. Modestes et populaires avant tout, ils proviennent de l'armée du Salut, d'Emmaüs ou directement du trottoir et sont choisis avec soin pour leur capacité à «dire l'humain ${ }^{5}$ » et à le raconter sur scène. Dans l'univers des spectacles de Jérôme Deschamps et Macha Makeïeff, les objets - malicieux, espiègles ou empotés - ont non seulement une vraie force comique mais également une véritable place dramatique. Déjà abîmés par la vie, ils ressemblent aux personnages qu'ils accompagnent. Un compagnonnage dramatique et poétique entre les acteurs et les objets se crée, dans un rapport quasi égalitaire. Sur scène, les objets acquièrent un pouvoir visuel et émotionnel qui leur permet de renseigner le public sur les habitudes, les troubles, les faiblesses et les addictions des personnages ainsi que sur leur statut social. Ils sont «l'assemblage minimum pour raconter le plus ${ }^{6} »$, écrit Macha Makeïeff.

Dans le chapitre de sa thèse ${ }^{7}$ consacré à la compagnie Deschamps-Makeïeff, Jean-Luc Mattéoli démontre comment l'objet scénique devient un véritable partenaire de jeu, «un provocateur du corps de l'acteur » qui entraîne des mouvements, «des attitudes », «des postures de l'excès [...]» et fait naître « le sentiment que les acteurs portent, avec les bouteilles de gaz, les planches, les lessiveuses, les bassines, les chariots ou les diables, une croix d'un genre nouveau ${ }^{8} »$. Le rapprochement qu'établit ici l'auteur entre ces objets du quotidien et l'objet religieux que représente la croix n'est pas anodin. En effet, dans les textes que Macha Makeïeff rédigent sur les spectacles de la compagnie, les termes religieux qu'elle emploie pour qualifier les objets sont nombreux. Les mots « reliques », « martyrs » ou encore « icônes ${ }^{9}$ » sont régulièrement employés par la metteure en scène et laissent deviner l'ampleur $\mathrm{du}$ culte qu'elle voue aux objets. Parallèlement aux spectacles, elle leur consacre des expositions aux tonalités mystiques dont la scénographie - à l'image d'une arche de Noé moderne - fait croiser les chemins d'une célébration funéraire et d'une fouille archéologique insolite.

En nous appuyant sur les recherches approfondies que Jean-Luc Mattéoli a effectuées dans ce domaine, nous tenterons d'éclairer la relation de la compagnie Deschamps-Makeïeff aux objets sous l'angle de la sacralité. À travers l'analyse des textes que Macha Makeïeff a consacrés aux spectacles de la compagnie ainsi qu'à ses expositions personnelles, nous interrogerons la manière dont l'objet, investi de cette sacralité, véhicule et incarne une certaine vision désenchantée de l'humain et du monde. Cette réflexion nous amènera à explorer la notion «d'objet sacré » en tant que réceptacle de ce désenchantement et générateur de spiritualité. Enfin, nous examinerons la manière dont les pratiques d'expositions de Macha Makeïeff relèvent à la fois d'une démarche salvatrice de la condition de l'objet et d'une réflexion sur le devenir d'une société et sur ce qu'il en reste.

\section{Rescapés, boîtes d'identités et traces d'humanité}

\footnotetext{
$4 \quad$ Terme fréquemment utilisé par Macha Makeïeff pour désigner les objets. Jean-Luc Mattéoli, L'objet pauvre, Mémoire et quotidien sur les scènes contemporaines françaises, PUR, Rennes, 2011, p.111.

6 Macha Makeïeff, Deschamps Deschiens, le théâtre de Jérôme Deschamps, op.cit., p. 60.

7 Jean-Luc Mattéoli, L'objet pauvre, Mémoire et quotidien sur les scènes contemporaines françaises, op. cit.

$8 \quad$ Ibid., p. 113.

9 Termes récurrents dans les ouvrages de Macha Makeïeff (voir par exemple L'Amour des choses ou Poétique du Désastre.)
} 
Dans sa thèse L'Objet pauvre, Mémoire et quotidien sur les scènes contemporaines françaises $^{10}$, Jean-Luc Mattéoli consacre un chapitre au cas de la compagnie DeschampsMakeïeff dans lequel il définit les objets comme «des contenants, sortes de «boîte[s] d'identité » des personnages dans lequel ils rangent leur «viatique ${ }^{11} »$. L'auteur ajoute que «le mot [viatique] n'aura jamais aussi bien convenu qu'à ces errants immobiles ${ }^{12}$ ». Il est intéressant de noter qu'au sens religieux du terme, le mot «viatique » désigne non seulement un soutien, mais aussi le sacrement de l'eucharistie administré à un mourant afin d'accompagner son passage de la vie terrestre à la vie éternelle. Dans l'antiquité grecque, le viatique représente la pièce de monnaie que l'on mettait dans la bouche des morts pour payer à Charon leur passage du Styx. Au sens où l'emploie Jean-Luc Mattéoli, le viatique qu'incarnent les objets pourrait symboliser le passage de l'acteur, du monde quotidien à l'espace sacré de la scène. Cette transition s'effectue en grande partie grâce aux objets scéniques sur lesquels les acteurs s'appuient pour construire leurs personnages. On peut en effet se demander ce que seraient Jérôme Deschamps sans sa poussette dans Les frères $Z_{\text {Zénith }}^{13}$, Yolande Moreau sans son cabas dans Les Pensionnaires ${ }^{14}$, François Morel sans son autoradio dans $C^{\prime}$ 'est magnifique ${ }^{15}$ ou encore Philippe Duquesne sans son Cubitainer de vin dans Les Pieds dans l'eau ${ }^{16}$ ? Ces quelques objets cités en exemple par Jean-Luc Mattéoli ${ }^{17}$ représentent particulièrement bien cette idée de «boîtes d'identités » que les objets symbolisent: par leur présence sur scène, ils assurent la transformation de l'acteur en personnage. Pourtant, ces objets sont des plus ordinaires : comment des objets aussi communs qu'un cabas ou un autoradio peuvent-ils exercer une influence sur l'identité et l'esthétique de la compagnie ? Selon Jean-Luc Mattéoli, « la première réponse, immédiate, est à chercher du côté de cette vie que les créateurs y pressentent ${ }^{18}$. » En effet, pour Macha Makeïeff, les objets constituent un véritable petit "peuple ${ }^{19}$ » dont les existences, vécues aux côtés des humains, ont fini par charger les objets d'humanité et de mémoire. Les objets usés semblent avoir une âme : tout comme les comédiens, ils intègrent la troupe avec leurs propres vécus qui leurs insufflent une charge émotionnelle toute particulière. Les lieux de leurs provenances marchés aux puces, brocantes, greniers - sortes de «cimetières exhibitionnistes » et d' " espaces de reliques pour gens ordinaire ${ }^{20} \gg-$ sont propices à libérer toutes sortes de récits. On les choisit abîmés, éclopés, parce que le mystère de leur aura - au sens benjaminien du terme ${ }^{21}$

$10 \quad$ Jean-Luc Mattéoli, L'objet pauvre, Mémoire et quotidien sur les scènes contemporaines françaises, op. cit., p.110.

$11 \quad$ Ibid.

$12 \quad$ Ibid.

13 Les Frères Zénith, spectacle de Jérôme Deschamps et Macha Makeïeff, création au théâtre municipal de Sète, le 25 janvier 1990.

14 Les Pensionnaires, spectacle de Jérôme Deschamps et Macha Makeïeff, création au théâtre national de Bretagne à Rennes, le 18 mai 1999.

15 C'est magnifique, spectacle de Jérôme Deschamps et Macha Makeïeff, création au théâtre de Nîmes, le 16 mai 1994.

16 Les pieds dans l'eau, spectacle de Jérôme Deschamps et Macha Makeïeff, création au théâtre de Nîmes, le 2 mars 1992.

17 Jean-Luc Mattéoli, L’objet pauvre, Mémoire et quotidien sur les scènes contemporaines françaises, op. cit., p. 110.

Jean-Luc Mattéoli, «L'objet pauvre dans le théâtre contemporain », Images Re-vues [En ligne], 4 | 2007, document 4, mis en ligne le 01 janvier 2007, consulté le 11 novembre 2011. URL : http://imagesrevues.revues.org/125

19 Phillipe Noisette, «L'ordre des choses », entretien avec Macha Makeїeff, in Beaux arts magazine $n^{\circ} 210$, novembre 2001, p. 56.

$20 \quad$ Henri Cueco, Le Collectionneur de collection, Seuil, Paris, 1995, p.128.

21 Voir à ce sujet Walter Benjamin, «L'Oeuvre d'art à l'ère de sa reproduction technique », in Euvres III, Paris, Gallimard, 2000, texte dans lequel Walter Benjamin développe son concept d' « aura » de l'œuvre d'art qui se matérialise par son unicité, son authenticité, sa présence « ici et maintenant », mais aussi son caractère 
- nourrit l'imaginaire et qu'ils deviennent ainsi des tremplins pour inventer des histoires.

Au delà de leur rôle de déclencheur narratif, de tels objets prennent une importance capitale au sein même de la direction scénique. L'objet devient le refuge qui permet de retenir le sens et le caractère concret de la réalité. Sa quotidienneté immédiate permet d'ancrer la gestuelle des acteurs dans «le vrai », de s'appuyer sur leur propre relation aux objets, et de trouver ainsi la bonne attitude, le bon jeu. Lorsque Yolande Moreau est interrogée au sujet de son rôle dans le spectacle Les Pieds dans l'eau, elle explique qu'une fois sur scène, elle se sent véritablement rassurée par sa «petite jupe » qu'elle «connaît bien », ses «petites chaussures» et sa vieille bassine ${ }^{22}$. La relation d'intimité et d'affection qu'elle entretient avec son trousseau l'aide à se sentir aussi à l'aise sur scène que dans le confort de sa "cuisine intérieure $^{23} \gg$. Le vêtement, en tant qu'objet identitaire, devient la peau du personnage. Il crée son identité et, par conséquent, représente une sorte de protection pour l'acteur. Chacun possède «son petit tas qui dit les fragilités, la détresse et l'exaltation du personnage ${ }^{24}{ }$. Chez les Deschiens, les comédiens semblent venir à la scène tels qu'ils sont à la ville. Il y a de l'humanité dans ces vêtements, la même humanité dont parle Roland Barthes à propos du parfait costume de théâtre :

«Il doit être une humanité, il doit privilégier la stature humaine de l'acteur, rendre sa corporéité sensible, nette et si possible déchirante. Le costume doit servir les proportions humaines et en quelque sorte sculpter l'acteur, faire sa silhouette naturelle, laisser imaginer que la forme du vêtement, si excentrique soit-elle par rapport à nous, est parfaitement consubstantielle à sa chair, à sa vie quotidienne; nous ne devons jamais sentir le corps humain bafoué par le déguisement ${ }^{25}$.»

L'utilisation de l'expression «consubstantielle à sa chair » dans le commentaire de Roland Barthes nous renvoie ici à une forme de sanctification du costume de théâtre. Pour l'acteur, le travail théâtral se réalise au contact de ces objets réels, porteurs d'histoires, d'imageries populaires et de mémoires. "Chaque objet doit avoir une fonction dramatique. [...] On doit privilégier la charge de violence qui fait l'objet précisément parce que son désastre est proche du nôtre ${ }^{26} \gg$ explique Macha Makeïeff. Ce type d'objet trouve donc son importance au sein de la compagnie dans le fait qu'il contienne en lui l'histoire des hommes. C'est précisément de cette humanité dont il est question.

Le choix de tels objets peut certainement trouver une seconde explication dans la capacité de l'objet à être un outil de communication visuelle instantané. Si plusieurs études ont déjà démontré comment le théâtre « sémantisait » tout objet ${ }^{27}$, d'une manière générale, un

sacré. La notion d"'aura" appliquée à l'objet ici reprend les mêmes caractéristiques.

22 Déclaration de Yolande Moreau lors d'une interview télévisée pour le journal de $20 \mathrm{~h}$ de France 3, diffusée le 15 juillet 1995 à l'occasion d'un reportage sur le spectacle Les pieds dans l'eau, festival d'Avignon.

$23 \quad$ Ibid.

24 Macha Makeïeff, Inventaire d'un spectacle, Les Pensionnaires, Arles, Actes Sud, 2000 , p.16 -17.

25 Roland Barthes, «Les maladies du costume de théâtre », in Ecrits sur le théâtre, Paris, Seuil, 2002, pp. 145-146.

26 Philippe Noisette, «L'ordre des choses », entretien avec Macha Makeïeff, art. cit., p. 56.

$27 \quad$ Voir à ce sujet les commentaires de Bertolt Brecht à propos de ses mises en scène ainsi que les études de Roland Barthes sur le théâtre, notamment «la sémantique de l'objet » in L'Aventure sémiologique, (Paris, Seuil, Points, 1985, pp. 249 - 260) et Ecrits sur le théâtre, (Paris, Seuil, 2002.) Dans L'Aventure sémiologique, Roland Barthes utilise l'exemple des commentaires de Brecht pour démontrer la sémantisation de l'objet (p. 254) : «Le commentaire le plus célèbre concerne la mise en scène de Mère Courage, où Brecht explique très bien le traitement long et compliqué qu'il faut faire subir à certains objets de la mise en scène, pour leur faire signifier tel concept ; car la loi du thêâtre, c'est qu'il ne suffit pas que l'objet représenté soit réel, il faut encore que le sens soit en quelque sorte détaché de la réalité : il ne suffit pas de présenter au public une veste de cantinière réellement usée pour qu'elle signifie l'usure ; il faut que vous inventiez, vous, metteur en scène, les signes de l'usure. » 
objet scénique n'est jamais choisi au hasard: il doit remplir une fonction esthétique, pragmatique et référentielle. Ainsi, le discours immédiat de l'objet permet d'appuyer la narrativité : grâce à lui, le spectateur découvre en un regard le lieu et le temps de l'action. Assurément, lorsque le spectateur voit un objet sur scène, il ne s'interroge guère sur sa valeur d'usage, mais il perçoit immédiatement, de manière plus ou moins consciente, le ou les systèmes de signes auxquels se rattache l'objet. Percevoir l'objet théâtral permet ainsi de comprendre dans un premier temps l'histoire des personnages, deviner leurs conditions sociales, leurs goûts, leurs savoir-vivre, leurs styles et leurs mentalités. Dans le minimalisme désertique des décors de la compagnie Deschamps-Makeïeff, les objets doivent être très signifiants pour être immédiatement interprétés par le spectateur. À ce titre, ils sont d'abord des marqueurs sociaux avant d'être des accessoires de théâtre. Ainsi, l'univers scénographique de la compagnie se compose d'espaces d'habitations réduits à des vestiaires industriels (Les Pieds dans l'eau) ou à un comptoir de zinc (C'est Magnifique, Les Frères Zénith), derrière lequel se dresse parfois un grand mur constitué de cageots ( $C$ 'est Magnifique). Or, d'après Francis Ponge, le cageot, constitué de façon à ce qu'on puisse le briser sans effort au terme de son usage, représente un objet « des plus sympathiques, - sur le sort duquel il convient toutefois de ne s'appesantir longuement ${ }^{28} »$. On aurait pu qualifier les personnages de la même manière. À travers ces exemples, on conçoit que le choix des objets n'est pas anodin : ils symbolisent et personnifient le destin des personnages. Chaque objet est en quelque sorte une allégorie du genre humain qui va permettre de dresser un état du monde et de la précarité humaine. Les objets choisis par Jérôme Deschamps et Macha Makeïeff bonbonnes de gaz, cabas, Cubitainer de vin, pichet en grès, bouillotte de vin rouge, seaux, bassines, casiers, chaises en formica, porte-bouteilles - dont la récurrence imprimera l'univers de La famille Deschiens, traduisent, en quelques secondes, le malaise brut d'un quotidien incommode.

Dans ce «monde rétréci ${ }^{29} »$ souvent représenté comme un «no man's land ${ }^{30}$ » inhospitalier, les personnages se livrent à un combat perdu d'avance, dans lequel les objets se présentent tantôt comme leurs béquilles, tantôt comme leurs plaies. La poussette de Jérôme Deschamps dans Les Frères Zénith offre ainsi des instants de consolation lorsqu'elle permet d'inviter à tout moment son partenaire à boire un verre, tandis que, dans Les pieds dans l'eau, le lit électrique de monsieur Saladin censé lui apporter un moment de relaxation se détraque et lui fait prendre les positions les plus inconfortables. Lorsque les objets s'obstinent à compliquer la vie des personnages en refusant de se soumettre à leurs attentes, ils traduisent l'échec de l'adaptation de l'homme à sa propre condition dans le monde :

«Le théâtre est forcément métaphorique, il s'agit d'un constat humain (et non sociologique [...]) qui parle de la difficulté des êtres aux prises avec le destin. Et le destin, c'est parfois un verre qui vous échappe, une inadaptation au pratique et au quotidien ${ }^{31}$. »

Si Jérôme Deschamps et Macha Makeïeff ne revendiquent nullement un quelconque « théâtre social », leur théâtre défend un point de vue sur le genre humain et la violence que la vie ordinaire inflige à tout individu. Malgré le comique des situations que les objets provoquent, on voit émerger la possibilité d'une dimension tragique, traduisant la circularité de l'existence de ces personnages, leur emprisonnement dans des schémas préétablis et leur difficulté d'en

\footnotetext{
28 Francis Ponge, Le Parti pris des choses, Paris, Gallimard, 1942, p. 38.

29 Macha Makeïeff, Inventaire d'un spectacle, Les Pensionnaires, Arles, Actes Sud, p. 9.

30 Jean-Luc Matteoli, L'objet pauvre, Mémoire et quotidien sur les scènes contemporaines françaises, op. cit, p. 121.

31 Propos de Macha Makeïeff relevés dans l'article « Mine de rien ? », entretien avec Jérôme Deschamps et Macha Makeieff, Sociétés \& Représentations, 2000/2 n 10, p. 252.
} 
sortir, nous ramenant à notre propre condition humaine. Le sentiment de désenchantement du monde qui traverse chaque spectacle semble se nourrir du déclin des valeurs censées participer au sens et à l'harmonie de l'existence humaine. Pourtant, l'historien des religions Mircea Eliade affirme que, «quel que soit le degré de désacralisation du Monde auquel il est arrivé, l'homme qui a opté pour une vie profane ne réussit pas à abolir le comportement religieux. On verra que l'existence même la plus désacralisée conserve encore des traces d'une valorisation religieuse du Monde ${ }^{32}$. » Chez les Deschamps-Makeïeff, on trouve les traces de cette valorisation à travers le traitement des objets.

\section{Icônes, reliques et talismans}

Nous avons pu nous apercevoir que le choix des objets de la compagnie DeschampsMakeïeff s'opère par une forme de personnification animiste qui nous conduit à explorer la notion d' «objet sacré » telle que nous la trouvons à plusieurs reprises dans les écrits de Macha Makeïeff. «Saintes de $\operatorname{carton}^{33} »$, «talismans de pacotille ${ }^{34} »$, « miséreux splendides $^{35} »$, «miracle miteux ${ }^{36} »$, les nombreux oxymores qu'elle utilise dans ses textes pour désigner les objets montrent que, malgré l'apparence insignifiante de leur existence, leur pouvoir est grand. En humanisant les objets de la sorte, Macha Makeїeff les investit d'une dimension spirituelle: ils sont à la fois les témoins et les martyrs du temps qui passe, menacés, comme l'homme, de disparition, et porteurs en eux-mêmes de la trace des êtres disparus. Pour comprendre comment une telle notion peut être attribuée aux objets dans le contexte de la compagnie théâtrale, il faut d'abord analyser la manière dont ils accèdent à cette forme de «sacralisation ». Chez Macha Makeïeff, le processus de sacralisation de l'objet engage tout d'abord une pratique de sauvetage ritualisée : "Aujourd'hui c'est aux objets assurément que François d'Assise aurait prêché sans fin, dans notre époque de gâchis organisé et tragique ${ }^{37}$ !» s'exclame-t-elle. D'une même insatisfaction profonde face à la vie naît le même besoin de se consacrer aux plus pauvres, aux plus démunis, aux plus marginalisés, car les objets font partie de ceux qui ne peuvent se défendre contre l'abandon et la maltraitance des hommes.

«Elles font mon paysage intérieur, ces choses, épinglées en moi, et c'est un droit d'asile que je réclame pour elle; le temps d'une célébration, j'arrache le singulier à la multitude clonée, à l'oubli collectif, cette dernière benne d'enlèvement. [...] Je les désire pour leur expressivité immédiate, je subis leur attraction, la terreur et le sublime des icônes, je les regarde comme des martyrs ${ }^{38} . »$

Le terme de «martyr » revient régulièrement pour désigner le peuple des objets dont Macha Makeïeff se fait protectrice. Le sens fortement religieux du terme évoque clairement l'image d'un peuple innocent en proie à la persécution. Avec compassion et charité, Macha Makeïeff part à «la chasse aux objets ${ }^{39} »$ comme elle partirait en croisade contre le gaspillage humain.

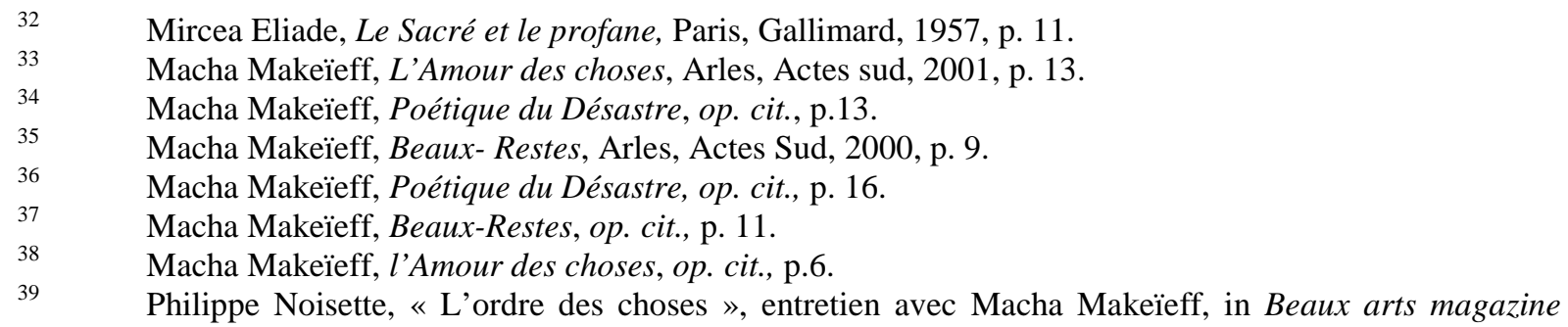


En apparence, il n'y a aucune consigne, aucune règle. Il faut «fouiller, retourner des tas de hardes, les ramasser un par un, ces restes de tenues, dans la rue, chez les autres, récupérer, les pendre, [...] et les apprivoiser ${ }^{40}$.» La seule consigne est d'avancer, à l'aveugle, "vers l'inattendu et ses surprises, vers le merveilleux, les lieux incertains de l'imaginaire », et d'en revenir «les mains sales, pleines de choses malcommodes, défaites, comme après un sac, un ravage, comme abandonnées par les casseurs ${ }^{41}$. » Le vocabulaire qu'elle utilise pour qualifier ce rituel donne l'impression qu'elle se livre à une véritable guerre sainte motivée par la volonté sincère d'extirper les choses d'un destin tragique. Ce combat, elle le poursuit depuis son enfance :

« Je me rappelle nos récoltes « de parterre » avec Georges trouveur magnifique et secret. Comme deux missionnaires on marchait jusqu'à s'épuiser, avec obligation de remplir le sac de toile, on scrutait le sol, le caniveau. Pas un mot, c'était la règle, seulement le rire ébloui au moment de la trouvaille, on ramassait frénétiquement avec le regard fixe du scrutateur, on grattait le bitume s'il fallait pour recueillir capsules, très petits débris [...], météorites tombées sur notre chemin, [...] tout ce qui scintillait. Revenus, on vidait le sac des minuscules tragédies ordinaires, sur la table, comme la cellophane des pièces à conviction. [...] Une histoire sans mots apparaissait, faits divers minuscules. Et la nuit qui suivait, dans le noir de la chambre, invisibles, on les sentait, les choses, dans leur immobile insomnie, la boite à chaussure glissée sous le lit comme un trésor de guerre ${ }^{42}$. »

Aujourd'hui, c'est toujours en «missionnaire », «en explorateur entêté », qu'elle se rend à ces «insolites rendez-vous ${ }^{43}$ ». Il y a comme une injonction divine à organiser cette étrange battue, et une fascination enfantine à imaginer les «minuscules tragédies ordinaires » que cristallisent ces objets. Tout se passe comme si les choses pouvaient ressentir, grâce à leur bienfaitrice, le soulagement après la violence de l'abandon. Si la recherche paraît incertaine, Macha Makeïeff sait reconnaître l'objet qu'elle attendait lorsqu'il se présente à elle. Le sentiment de surprise de la trouvaille est vite remplacé par l'impression de se trouver face à l'objet convoité, dont la cohérence apparaît rapidement. «Je sais que le hasard n'a rien à y faire, il y a comme prédestination ${ }^{44}{ }$ explique-t-elle. Les allures d'odyssées mystérieuses que prennent ses quêtes ne sont pas sans rappeler l'esprit du mouvement surréaliste, particulièrement lorsqu'elle déclare que ses récoltes sont "l'équivalent de ce qui se passe dans le rêve, on avance, étourdi, captif, vers ce qui est une promesse ${ }^{45}$. » On trouve, en effet, chez André Breton, cette même idée selon laquelle la trouvaille d'objets joue un rôle de catalyseur et remplit la même fonction que le rêve : «elle libère l'individu de scrupules affectifs paralysants, le réconforte et lui fait comprendre que l'obstacle qu'il pouvait croire insurmontable est franchi ${ }^{46}$. » La quête fait place alors à la trouvaille : moment magique où découvertes paraissent être le fruit du hasard, et pourtant, leurs trouvailles respectives correspondent à des besoins inconscients, des désirs particuliers. En effet, les objets trouvés sur ce marché - un masque pour Giacometti et une cuillère en bois dont le manche est décoré d'un soulier miniature pour Breton - répondent à une certaine nécessité chez chacun. Pour Giacometti, ce masque permettra de dépasser un blocage et de résoudre un problème plastique. Quant à Breton, la cuillère-soulier viendra combler le désir, survenu quelques mois auparavant, de posséder un cendrier en forme de pantoufle censée rappeler celle perdue par Cendrillon. Avec
} 
l'on reconnaît, comme une évidence, la coïncidence parfaite de l'objet et de ce qu'il portait en lui, sans même le savoir encore. "C'était un objet complètement différent de l'autre » disait Kantor, un objet unique et irremplaçable malgré son apparente banalité.

Nous pouvons donc affirmer que cette dévotion pour les objets n'est pas neutre dans l'univers de la compagnie, elle en fait partie intégrante. Elle est la gardienne de l'aura mystérieuse qui enveloppe chaque accessoire sur scène et qui rend chacun d'entre eux si particulier. Cependant, cette dévotion - qui semble principalement liée à la personne de Macha Makeïeff ${ }^{47}$ - ne peut tenir ni aux objets eux-mêmes, ni à la fin pour laquelle ils ont été fabriqués. Elle réside dans le monde imaginaire qui entoure l'objet et qui lui donne une valeur bien supérieure à celle que suscite sa simple fonction utilitaire ou esthétique. Il s'agit d'une projection. Cette valeur ajoutée joue un rôle très important dans la sacralisation des choses. Pour analyser ce phénomène, il est intéressant de se pencher sur le «concept du sacré ${ }^{48}$ » au sens où Mircea Eliade le définit. Dans ses écrits, Eliade revendique la relation au sacré comme une donnée universelle et immuable de notre humanité. En d'autres termes, seule l'expérience du sacré permettrait de développer la conscience de l'existence :

« La conscience d'un monde réel et significatif est intimement liée à la découverte du sacré. Par l'expérience du sacré, l'esprit humain a saisi la différence entre ce qui se révèle comme étant réel, puissant, riche et significatif, et ce qui est dépourvu de ces qualités, c'est-à-dire le flux chaotique et dangereux des choses, leurs apparitions et disparitions fortuites et vides de sens ${ }^{49}$.

Pour Mircea Eliade, tout être humain serait animé par le désir permanent de construire sa propre expérience du sacré de manière à trouver des réponses aux doutes qui nous envahissent et atténuer ainsi les angoisses que notre propre existence nous inflige. De la même façon, le besoin irrésistible de la compagnie Deschamps-Makeïeff de construire son expérience du sacré à travers les objets dévoile une quête du sens de l'humanité doublée d'une lutte désespérée contre le désenchantement du monde. C'est cette même problématique que nous retrouvons au cœur de chaque spectacle et de chaque exposition. $\mathrm{Si}$, en apparence, la cause des objets semble plus juste que celle des hommes, elle traduit surtout, à nouveau, la colère éprouvée devant le constat de la défaite de l'humain. Lorsque Dieu, déçu par le comportement des hommes, ordonne à Noé de construire une arche afin de préserver les animaux innocents du déluge, Macha Makeïeff tente d'une manière similaire de protéger les objets de l'incohérence du monde qui les entoure en leur offrant «l'asile » que peut représenter le théâtre ou les expositions. Largement inspirée de ses élans spirituels, la démarche artistique de Makeïeff se nourrit de cette pratique de sauvetage de l'objet afin de lui donner un peu d'éternité et de retarder sa disparition. Comme dans les spectacles, Macha Makeïeff entretient cette volonté d'arracher l'objet à son destin d'exclu et de malmené pour lui donner une chance de briller sous la lumière :

«Une rédemption sensuelle et un peu de recueillement pour vanités et merveilles, célébration sans épouvante, le surnaturel dans le quotidien. Rechercher la beauté, celle des mis au rebut, partout, par terre et en haut des murs, collée, jaunie, détrempée, déchirée, répandue, meurtrie. Je la traque. En attente - toutes ses traces sont mes espoirs. Elle saurait nous sauver car tout part à l'oubli ${ }^{50}$. »

\footnotetext{
cette cuillère, le cendrier dont il avait rêvé a pris un autre aspect, mais répond à son désir.

$47 \quad$ C'est à Macha Makeïeff que revient la tâche de trouver les objets nécessaires aux spectacles.

$48 \quad$ Voir les différents ouvrages que l'auteur consacre au sujet, notamment La nostalgie des origines, Le

Sacré et le Profane ou encore Histoire des croyances et des idées religieuses.

$49 \quad$ Mircea Eliade, Histoire des croyances et des idées religieuses, Paris, Payot, 1989, p. 7.

50 Macha Makeïeff, Beaux-Restes, op. cit., p. 21
} 
Cette «traque » serait-elle alors une forme de recherche du salut? Uniquement composées d'objets, les expositions de Macha Makeïeff se présentent comme des récits silencieux, des contes immobiles. Il s'agit de «jouer les archivistes» en présentant les fouilles d'une « extravagante archéologie ${ }^{51}$ ». Les expositions comme L'Amour des choses $^{52}$, Beaux-Restes ${ }^{53}$ ou encore Bêtes royales et objets perdus ${ }^{54}$ sont des invitations à errer parmi des objets «morts» (poupée borgne, lion empaillé, chemise sous verre...) et nous donne l'occasion de les reconsidérer : « une façon de réfléchir à la présence de ces objets autour de nous, à la force de désir qu'ils contiennent encore. Je tente ainsi de parler de nos désastres minuscules et de cette douleur exquise qui nous tient en vie quand même ${ }^{55}$ ». À travers ses expositions, Macha Makeïeff nous propose donc de regarder, au cœur du délabrement de ces choses, ce qui nous reste et résiste encore. Bien que les objets présentés restent des reliques fictionnelles, ils sont utilisés comme des allégories de l'homme et de son destin. Il s'agit toujours d'œuvrer contre le désenchantement du monde et sa dégradation. Si le projet existentiel rejoint celui qu'elle mène aux côtés de Jérôme Deschamps au théâtre, la forme de l'exposition change radicalement le statut de l'objet et participe au phénomène de sacralisation tel que nous l'avons développé. Magnifié par les lumières, valorisé par les vitrines et la scénographie, le temps semble s'arrêter sur l'objet. Rendu inaccessible à la manipulation, le changement de contexte que lui offre l'exposition lui donne une importance dont il serait autrement dénué. Le regard bascule alors dans une forme de contemplation qui valorise à son tour l'objet exposé en lui donnant l'aura d'une icône. Le philosophe Krzysztof Pomian décrit ce phénomène sous le terme de «sémiophore». Le terme de « sémiophore » désigne les objets investis de significations culturelles, sociétales et narratives, qui deviennent signifiants lors d'une opération de mise en valeur :

«[...] tout objet devient sémiophore à la suite de la décontextualisation et de l'exposition. Et il le reste aussi longtemps qu'il est exposé. Il en est ainsi parce que placer un objet, quel qu'il soit, dans une vitrine, dans un album, dans un herbier, sur un socle, le suspendre au mur ou au plafond, le séparer par une clôture, une barrière, un cordon, un grillage ou simplement par une ligne dessinée, à ne pas transgresser, le faire surveiller par un gardien ou mettre à côté un panneau avec l'interdiction de s'en approcher et surtout de le toucher, tout cela revient à imposer à des personnes qui se trouvent alentour l'attitude des spectateurs, à les inciter à se tourner vers cet objet et à arrêter sur lui le regard. Et cela contribue à attirer l'attention sur cet objet et montrer que la contemplation en change celui qui le fixe, car elle lui apporte quelque chose dont il serait autrement dépourvu ${ }^{56}$. »

Nous pourrions aller jusqu'à dire que cet état de contemplation change autant celui qui fixe que ce qui est fixé, car c'est uniquement lors de cet échange que l'objet se charge d'une signification dont il serait autrement également démuni. Par cette opération de valorisation, Macha Makeïeff parvient à offrir à ses protégés un moment de répit, tout en donnant à réfléchir sur l'humain et la société contemporaine.

$51 \quad$ Macha Makeïeff, Le Grand Ordinaire et le petit ménager, les collections Deschamps 1973-1993, catalogue de l'exposition de La Villette (7 avril-14 juin 1992 ; 1er décembre 1992-3 janvier 1993), Paris, 1992, p.11.

L'Amour des choses, exposition au Théâtre National de Chaillot, Paris, 2001. Scène Nationale d'Annecy, Bonlieu, 2002. Comédie de Genève, 2003.

$53 \quad$ Beaux-Restes, exposition au Musée des Arts Décoratifs, Paris, 2000.

$54 \quad$ Bêtes royales et objets perdus, exposition au Musée du Louvre, Paris, 2005.

55 Macha Makeïeff, propos recueillis à l'occasion de l'exposition L'Amour des choses, disponibles en ligne : [http://www.tsr.ch/emissions/archives/faxculture/1256797-vincent-delerm-et-mathieu-boogaerts-dans-lacour-des-grands.html] consulté le 13 novembre 2011.

$56 \quad$ Krzysztof Pomian, Sur l'histoire, Gallimard, Paris, 1999, p. 215 
Si, selon la formule de Roland Barthes, «l'objet est la signature humaine du monde ${ }^{57} »$, dans l'univers de la compagnie Deschamps-Makeïeff, l'objet se conçoit comme une «allégorie de nos destins cassants comme du plastique ${ }^{58}$ ». Véritable porte-parole de la vie des hommes, il est systématiquement exploité pour la sensibilité de sa force sémantique, truffée d'indices psychologiques, sociologiques et poétiques. Le théâtre, tout comme l'exposition, le dotent d'un certain pouvoir spirituel qui dépasse sa valeur objective. Accessoiristes, metteurs en scène, comédiens : tous s'appliquent à charger ces objets de vie, d'histoires, d'expressions et de langage signifiant pour leur confier les rôles d'authentiques actants, dont le jeu dramaturgique doit égaler celui des acteurs. Source d'inspiration, déclencheur narratif, viatique de l'acteur, l'objet devient ainsi un véritable partenaire, un compagnon aux airs de chien mal dressé dont les situations tragicomiques déclenchent, malgré elles, des rires sauvages, grinçants et teintés d'amertume. On rit de la poussette qui fume, de la bouteille de gaz qui éclate, de la télévision qui grille, de la machine à sous qui se détraque, des bouteilles volantes, de tout ce qui «glisse, se renverse, échappe, craque et se disperse $^{59} »$. On rit de ces choses parce qu'elles sont les marques de notre propre condition, et qu'elles présentent l'avantage de nous remettre à notre place de «petits propriétaires désabusés ${ }^{60} \gg$.

Dans la guerre sainte contre le désenchantement du monde à laquelle se livre la compagnie, les objets se présentent comme des boucliers. Mais il faut savoir lire, derrière la puissance sacrée que l'on veut bien leur prêter, l'expression d'une impuissance infinie : celle de l'homme face à son destin. Telle une nouvelle forme de croyance, la sacralisation de l'objet pratiquée au sein de la compagnie se construit comme une autarcie - voire comme un système cosmique à part - qui s'administre par ses seules lois et prend une signification personnelle et intime, propre à l'univers si particulier de la compagnie. Libéré de sa dimension religieuse, l'objet sacré se donne à penser sous l'angle du poétique tel que le propose le philosophe Jean-Jacques Wunenburger :

« Ne gagnerait-on pas en fin de compte à dégager la sacralité de son contexte religieux, même s'il est omniprésent, pour le considérer dans sa donation pure qui ne se trouve nulle part mieux que dans le poétique ? Le verbe poétique le capte dans les replis du langage qui assure une sorte de retentissement émotionnel et imaginaire, libre d'autres croyances particulières. Le poète est peut-être celui qui délivre le sacré de toute sa gangue à la fois trop divine (en le déliant des dieux d'une religion) et trop humaine (en le dégageant de pratiques visant des pouvoirs). Cette vocation du poétique à appréhender le sacré est peutêtre plus qu'un fait contingent, mais l'essence même du poétique, comme l'essence du sacré serait de poétiser l'existence ${ }^{61}$. »

Poétiser l'existence en se faisant l'explorateur de son propre territoire. Observer ses objets familiers, devenus soudainement exotiques. En isoler quelques-uns, les plus significatifs et les épingler comme on le ferait d'un insecte rare et précieux, ou les conserver sous vitrine comme des traces archéologiques, des témoignages inestimables de la vie des gens. Telles sont les expériences que les spectacles de la compagnie Deschamps-Makeïeff - autant que les

\footnotetext{
57 Roland Barthes «Les planches de l'Encyclopédie », in Le Degré zero de l'écriture, Seuil, Paris, 1953, p. 90 .

Macha Makeïeff, L'Amour des choses, op. cit., p.13.

Macha Makeïeff, Deschamps Deschiens, le théâtre de Jérôme Deschamps, op. cit., p. 27.

60 Macha Makeïeff, Au Chic Deschiens, op. cit., p. 10.

61 Jean-Jacques Wunenburger, Le Sacré, Paris, PUF, 1981, p. 121.
} 
expositions de Macha Makeïeff - sont susceptibles de nous faire vivre. Plaisir et surprise des détournements, magie du dérisoire, poésie du quotidien, les objets magiques du théâtre de Jérôme Deschamps et de Macha Makeïeff fonctionnent avec leurs propres règles. On se surprend à se laisser atteindre par les choses et on s'amuse à regarder les gens, nous-mêmes, comme si c'était la première fois. L'autodérision - arme secrète pour tenter de réenchanter provisoirement le monde - prend alors le pas sur le dérisoire pour profiter, le temps d'une représentation, «d'un instant de sublime et de banal ${ }^{62}$ ».

\section{Bibliographie}

Barthes Roland, « la sémantique de l'objet » in L'Aventure sémiologique, Paris, Seuil, Points, 1985.

Barthes Roland, «Les maladies du costume de théâtre », in Théâtre populaire ${ }^{\circ} 12$, marsavril, 1955.

Barthes Roland, «Les planches de l'Encyclopédie », in Le Degré zéro de l'écriture, Seuil, Paris, 1953.

Barthes Roland, Ecrits sur le théâtre, Paris, Seuil, Points, 2002.

Barthes Roland, Essais critiques, Paris, Seuil, Points, 1981.

Benjamin Walter, «L' Euvre d'art à l'ère de sa reproduction technique », in Euvres III, Paris, Gallimard, 2000.

Breton André, L'Amour fou, Paris, Gallimard, 1937.

Cueco Henri, Le collectionneur de collection, Seuil, Paris, 1995.

Eliade Mircea, Histoire des croyances et des idées religieuses, Paris, Payot, 1989.

Eliade Mircea, Le Sacré et le profane, Paris, Gallimard, 1957.

Makeïeff Macha, "L'ordre des choses », entretien par Phillipe Noisette, in Beaux art magazine $\mathrm{n}^{\circ} 210$, novembre 2001.

Makeïeff Macha, Au chic Deschiens, Paris, Seuil, 1997.

Makeïeff Macha, Beaux-Restes, Arles, Actes Sud, 2000.

Makeїeff Macha, Deschamps Deschiens, le théâtre de Jérôme Deschamps, Paris, Séguier, 1989.

Makeïeff Macha, Inventaire d'un spectacle, Les Pensionnaires, Arles, Actes Sud, 2000.

Makeїeff Macha, L'Amour des choses, Arles, Actes Sud, 2001.

Makeïeff Macha, Le Grand Ordinaire et le petit ménager, les collections Deschamps 19731993, catalogue de l'exposition de La Villette (7 avril-14 juin 1992 ; 1er décembre 1992-3 janvier 1993), Paris, 1992.

Makeïeff Macha, Poétique du Désastre, Arles, Actes Sud, 2001.

Mattéoli Jean Luc, L'Objet pauvre, Mémoire et quotidien sur les scènes contemporaines françaises, PUR, Rennes, 2011.

" Mine de rien ? », entretien avec Jérôme Deschamps et Macha Makeïeff, Sociétés \& Représentations, 2000/2 n 10, p. 252.

Noisette Philippe, «L'ordre des choses », entretien avec Macha Makeïeff, in Beaux arts magazine $n^{\circ} 210$, novembre 2001.

Pomian Krzysztof, Sur l'histoire, Gallimard, Paris, 1999.

Ponge Francis, Le Parti pris des choses, Paris, Gallimard, 1942.

Wunenburger Jean-Jacques, Le Sacré, Paris, PUF, 1981. 


\section{Pour citer le document}

Camille Fosse, «La force des choses», Agôn [En ligne], Dossiers, №4 : L'Objet, Objet, mémoire, identité, mis à jour le : 20/12/2011, URL : http://agon.enslyon.fr/index.php?id=1964. 